\title{
Sperm count and future challenges for cancer patients
}

\begin{abstract}
Cancer has significantly harmful effects on sperm count, motility and sexual life of the survivor, thereby adversely affecting the fertility and post cancer quality of life. Use of safer chemotherapeutic agents, protection from radiation damage, cryopreservation of sperm and testicular tissue and use of protective drugs to reduce testicular damage is recommended. More research is needed to safeguard fertility of cancer affected children as testicular tissue cryopreservation is the only fertility preservation option. Advanced understanding of in vitro sperm production is needed. In this era of advanced assisted reproduction, the minimum requirement for fertilization is one healthy sperm for one oocyte. Children born after chemotherapy do not have statistically significant increase in malignant neoplasms. Proper cancer counseling and referral for fertility preservation are of high importance to protect fertility. The focus of this review is to share knowledge of sperm formation, importance of sperm count, nature of damage to male fertility, remedies to overcome damage and to improve post cancer quality of life of male cancer survivors.
\end{abstract}

Volume II Issue 3 - 2020

\author{
Murid Javed \\ Origin Elle Fertility Clinic \& Women's Health Centre, Canada
}

Correspondence: Murid Javed, OriginElle Fertility Clinic \& Women's Health Centre, Ottawa, Canada, Tel + | 4167319885, Email murid.javd@gmail.com

Received: April 15, 2020 | Published: June 05, 2020

Keywords: fertility, cancer, sperm, cryopreservation, chemotherapy, radiotherapy

\section{Sperm origin}

The spermatozoon (one sperm) is a mature male germ cell with one half of the normal number of chromosomes of any species. It is produced in the seminiferous tubules of the testes and fertilizes the mature ovum containing one half of the number of chromosomes. It is microscopic in size, looks like a translucent tadpole, and has a flat elliptical head containing a spherical center section, and a long tail by which it propels itself with a vigorous lashing movement (Figure 1A).

The semen, in addition to sperm, consists of secretions from the testes, seminal vesicles, prostate, and bulbourethral glands. The spermatogonia (Precursor cell of sperm) contribute by producing millions of sperm, while the Sertoli cells secrete fluid to act as a suspension for the transport of sperm from the seminiferous tubules and genital ducts. ${ }^{1}$ Different parts of the male urogenital system are shown in Figure 1B. The testes are composed of numerous seminiferous tubules, where sperm formation takes place. The seminiferous tubules mainly contain; spermatogonia which are stem cells of sperm, Sertoli cells and Leydig cells which produce testosterone (Figure 1C \& 1D). After production, sperm are stored in epididymis and at the time of ejaculation, pass through the vas deference and urethra. Major volume of the semen is contributed by the seminal vesicles and prostate gland. The cellular component of the semen should only contain sperm. However, occasionally, other cells generally called round cells, like epithelial cells, immature germ cells and white blood cells are also observed. The presence of non-sperm cells, in semen may be indicative of testicular damage (if immature germ cells are present), pathology of the efferent ducts or inflammation of the accessory glands (if leukocytes are present). The acceptable limit of round cells in the semen is up to $1 \times 10^{6}$ cells per $\mathrm{ml}$.

\section{Significance of sperm count}

The normal lower reference limit for human sperm concentration is 15 million $\left(15 \times 10^{6}\right)$ spermatozoa per $\mathrm{mL}$ and the lower reference limit for the total sperm number is $39 \times 10^{6}$ spermatozoa per ejaculate. ${ }^{2}$ In natural conception only a few sperm reach the ampulla or the site of fertilization ${ }^{3}$ indicating that other are either lost in female reproductive tract or expelled after coitus. In intrauterine insemination procedure (IUI), 5million motile sperm are recommended for optimal conception. In the in vitro fertilization (IVF) procedure, generally 100,000 motile sperm are added per one $\mathrm{mL}$ of media and in the intra-cytoplasmic sperm injection (ICSI) procedure only one sperm is injected into each egg. Even in nature only one sperm fertilizes the egg. The option of conception, IUI, IVF or ICSI is mainly selected based on the number of progressively motile sperm in the ejaculate unless there is any other female factor infertility.

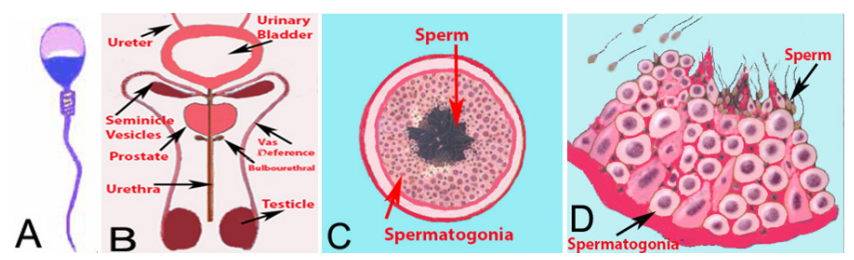

Figure I (A) Diagrammatic presentation of a sperm, (B) Diagrammatic presentation of male urogenital system. The sperm are produced in the tubules of testicle. The ejaculate consists of fluid produced by testes, seminal vesicles, prostate and bulb urethral glands. (C) A cross section of a tubule. Each testicle is composed of numerous tubules. (D) A magnified diagram of a section of the tubule. The spermatogonial cells are round but the mature sperm take the shape of a tadpole.

The sperm concentration in the ejaculate varies from person to person and even in 2 ejaculates of the same person. It is therefore, recommended to report the total number of spermatozoa per ejaculate, as this parameter provides a measure of the capability of the testes to produce spermatozoa and the patency of the male reproductive tract. If no sperm is found in the ejaculate, centrifugation is performed to concentrate the sperm in the pellet. However, if no spermatozoa are observed in the sediment of the centrifuged ejaculate, azoospermia (no sperm) can be suspected. It may not be accurate to declare azoospermia based only on one ejaculate as sperm may be found in another ejaculate. Also, the centrifugation of ejaculated sample at $3000 \mathrm{~g}$ for 15 minutes does not pellet all spermatozoa from an 
ejaculate. The author recommend diluting the ejaculate with Hepesbuffered media ( 1 part semen and 1 part media) before centrifugation and examining at least 3 ejaculates at weekly intervals to declare azoospermia.

\section{Other important sperm parameters}

In addition to the sperm count, other parameters like sperm vitality, motility, morphology and composition of the seminal fluid are important for normal sperm function. The progressive sperm motility is related to pregnancy rates. The sperm motility should be assessed as soon as possible after liquefaction of the sample, preferably 30 minutes after ejaculation or within 1 hour following ejaculation, to limit the deleterious effects of dehydration, $\mathrm{pH}$ or changes in temperature. Each ejaculate contains motile and immotile spermatozoa at variable ratio.

\section{Control of sperm production}

The follicle stimulating hormone (FSH) and leutinizing hormone (LH) are produced by the anterior pituitary gland in response to stimulation by gonadotropin releasing hormones (FSH RH, LH RH) from the hypothalamus (Figure 2). The FSH induces expression of LH receptors on Leydig cells. The LH in turn stimulates the Leydig cells in the testicular interstitium to synthesize and secrete testosterone which supports spermatogenesis in the germinal epithelium and sperm maturation in the epididymis. The Sertoli cells produce inhibin B after stimulation by FSH. The secretion of inhibin B and testosterone results in a negative feedback to the pituitary and hypothalamus, thereby reducing the secretion of the pituitary gonadotropins. The healthy male hypothalamic-pituitary-gonadal axis controls two functions that are essential for male reproduction: the production of appropriate quantities of testosterone and the generation of healthy mature sperm capable of fertilizing an oocyte.

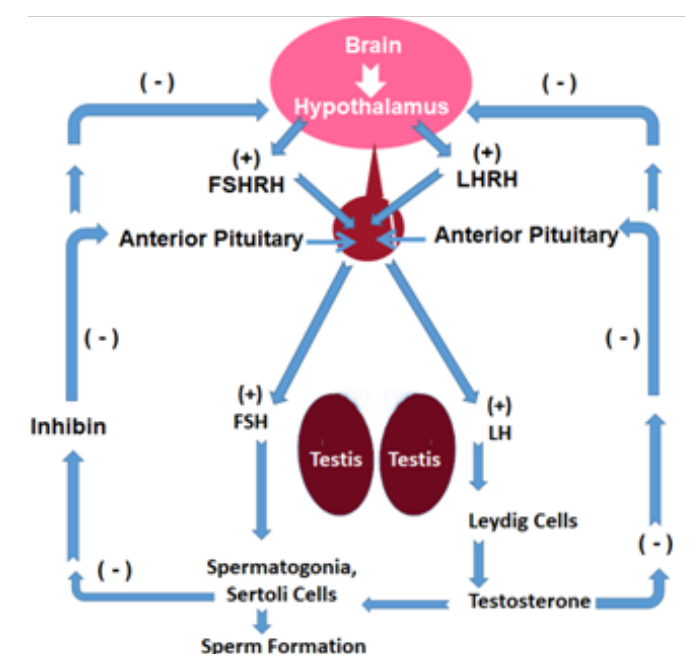

Figure 2 Diagrammatic presentation of endocrine pathways for regulation of sperm production.

\section{Effects of cancer on sperm count and patient's sexual life}

Cancer and its treatment may put survivors at risk of temporary or permanent infertility. The radiation and chemotherapy kill actively dividing cells. The cancer cells divide much more actively than most other body cells, so they are killed. In males, sperm cells also divide actively; therefore, they are also likely to be killed by chemotherapy and radiation. An increasing number of young survivors suffer subfertility or infertility after cancer treatment. Therefore, the impact of cancer treatment on future fertility of adolescent patients is of significant concern, both to the patients and their parents. ${ }^{4}$

The side effects of adolescent cancer treatment also include subsequent development of erectile dysfunction and hypogonadism in adulthood. ${ }^{5}$ The erectile dysfunction may be due to physiological and psychological changes due to diagnosis of cancer and its treatment. The hypogonadism can arise because of testicular radiation, chemotherapy or pituitary insufficiency after brain radiation or surgery. The hypogonadism results in low testosterone and poor sperm count in adolescent testicular cancer survivors, thus cause a negative effect on quality of sexual life. The childhood cancer survivors who are treated with chemotherapy and/or testis irradiation are at risk for gonadal dysfunction, independent of the age at which the cancer treatment is administered. ${ }^{6}$ The germinal epithelium is particularly sensitive to direct testicular radiation or by scatter radiation resulting from radiotherapy of pelvis, bladder and prostate. More than $50 \%$ of boys surviving childhood cancer are diagnosed with some degree of dysfunction of spermatogenesis. ${ }^{5}$

The recovery of spermatogenesis in long-term survivors of childhood cancer depends on the type of cancer, type and duration of treatment and the organ involved. Azoospermia and oligospermia are common long-term side effects in male childhood Hodgkin's lymphoma patients after radiotherapy and chemotherapy, especially when alkylating agents, e.g. mustine or procarbazine, are used. ${ }^{6}$ After chemotherapy, sperm production slows down or may stop altogether. Some sperm production usually returns in 1 to 4 years, but it can take up to 10 years. If sperm production has not recovered within 4 years, it's less likely to return. Regular follow up visits to determine spermatogenesis, sexual function and psychological wellbeing are recommended.

Difficulty achieving a pregnancy can certainly affect the quality of life for the patients and their families. These patients, therefore, should be followed up in an organized manner to address these issues after their treatment because infertility symptoms may not arise until many years after the cancer treatment. It is best that physicians discuss these risks with cancer patients before cancer treatment begins. The Ethics Committee of ASRM recommends that clinicians should inform patients receiving potentially gonadotoxic therapies about options for fertility preservation and future reproduction prior to the initiation of such treatment. ${ }^{7}$ All available options should be offered and can be performed alone or in combination, often without causing significant delay to cancer treatment.

\section{Strategies to prevent cancer damage}

Sperm cryopreservation: Sperm cryopreservation is the gold standard for maintaining fertility in male survivors of cancer. It is highly recommended for all males regardless of age and type of cancer. The frozen semen maintains the reproductive potential indefinitely while stored at the temperature of liquid nitrogen $\left(-196^{\circ} \mathrm{C}\right) .^{8}$ The other options are gonadal transposition, and testicular tissue cryopreservation and re-implantation. ${ }^{9}$ It is imperative to preserve the fertility of male cancer patient, as it is a basic human right. ${ }^{10}$

Successful pregnancies and deliveries have been reported after use of cryopreserved semen from cancer patients. ${ }^{11,12}$ It is recommended to obtain 2-3 ejaculates per patient before cancer treatment because sperm concentration, motility and quality of sperm are usually poor in cancer patients. The abstinence period of 48 hours is appropriate. ${ }^{13}$ In 
emergency situations, if possible semen samples can be cryopreserved after 24 hours of abstinence or even 2 ejaculates on the same day can be frozen.

The laboratories should decide the number of cryovials/straws based on the sperm concentration and motility. Efforts should be made to maximize the cryovials/straws as multiple units can be thawed if more sperm are needed. The repeated thawing and refreezing sperm will compromise their viability. ${ }^{14,15}$ With the advent of ICSI, one normal sperm per oocyte is needed and using cryopreserved spermatozoa, success rates are comparable to standard IVF and ICSI procedures in infertile couples. ${ }^{16}$ The idea is to preserve enough sperm for future procedures to meet future family needs. Usually semen samples from cancer patients are compromised and generally not suitable for natural conception or IUI. In such cases ICSI is the only choice.

If ejaculation is not possible for the patient, electro-ejaculation, penile vibratory stimulation and search for spermatozoa in urine samples or in tissue retrieved by testicular sperm extraction (TESE) are useful alternatives. ${ }^{16} \mathrm{~A}$ post-ejaculation urine sample, to rule out retrograde ejaculation, is easy to collect and is less invasive. The sperm cryopreservation provider should train the staff in communicating/managing the adolescent cancer patients and their families about sperm cryopreservation and increase utilization of fertility preservation referrals to increase provision of service to males at risk before cancer treatment initiation. ${ }^{17}$ The staff must be knowledgeable about the infertility risks associated with cancer therapies and proactively address fertility with all patients who might one day wish to have a child. ${ }^{18}$

\section{Testicular tissue cryopreservation}

Semen retrieval is not possible in pre-pubertal boys and may not always be possible in adolescents. In such cases, cryopreservation of testicular tissue (Isolated spermatogonial stem cells) is a viable means of preserving fertility. ${ }^{19}$ However, still there is challenge in pre-pubertal male's testicular tissue cryopreservation. Although spermatogonial cells are present but mature spermatozoa are absent. In such cases testicular tissue preservation is needed to safeguard the best interests of the child. ${ }^{4}$ The author recommends that this option should be offered to pre-pubertal patients as scientific progress in this field is encouraging and in vitro spermatogenesis from spermatogonial cells may become a reality. To minimize trauma to the patients, testicular tissue extraction should be combined with other interventions requiring anesthesia, such as bone marrow sampling. ${ }^{20,21}$

Overall long-term survival of childhood cancer has steadily increased up to about $80 \%$, owing to optimized treatment regimens, ${ }^{6,22}$ therefore, every possible option must be considered for fertility preservation. For adult patients with non-obstructive azoospermia, severe oligozoospermia, necrozoospermia or ejaculation disorders, TESE is often the only acceptable means of tissue/sperm retrieval for fertility preservation. ${ }^{22}$

\section{Use of drugs to reduce testicular damage}

Another option, although still experimental, to prevent testicular damage, is the use of drug therapies that reduce the gonadotoxic impact of chem- or radiotherapy. Co-administration of the immunemodulating compound AS101 during cyclophosphamide treatment appears to provide protection. ${ }^{22}$ Also use of precision medicine which takes into account the influence of individuals' genes, environment and lifestyle exposures to tailor interventions is possible. ${ }^{23}$ Some biomarkers are increasingly capable of distinguishing genotypic or phenotypic traits of individual tumors, and are being linked to the selection of treatment protocols. For example platinum derivatives are commonly employed against solid tumors that kill cells by covalent attachment to DNA. ${ }^{24}$

\section{Effect of testicular cancer on fertility}

Fertility may be poor during the two years before testicular cancer is discovered. Although only 1 to 3 percent of men with testicular cancer get cancer in both testicles, the cancer-free testicle may not be totally normal. Testicular germ cell tumors, account for more than $97 \%$ of testicular cancers. Almost all testicular germ cell cancers are treated with surgery to remove the affected testicle, followed by radiation or chemotherapy. The 15 -year survival rates for testicular cancer are $95 \%$. Testicular cancer patients often face sexual and fertility problems after treatment. The men with bilateral testicular removal require lifelong testosterone supplementation. ${ }^{25}$ Most men with one healthy testicle may produce sufficient male hormones and sperm to continue sexual relations and father children, however, sperm banking is highly recommended prior to treatment.

\section{Quality of life and future challenges for male cancer survivors}

The quality of life may decline significantly during cancer treatment and may remain low for a variable period after cure. A significant number of cancer patients report lower overall physical well-being than their peers. Many survivors also suffer from a fear of recurrence of cancer. ${ }^{25}$ Also, patients experience a loss of support due to reduced contact with medical professionals. A good case management can improve quality of life and ease re-entry to normal life. ${ }^{26}$

Cancer survivors may experience psychological distress due to effects of cancer treatment on their reproductive and sexual function and intimate relationships. Assessment of psychological health should be a key part of long term follow-up, and provision should be made for specialist psychological input where indicated ${ }^{27}$ Although the negative psychosocial impact of cancer-related infertility is now well accepted, there is need to explore how to improve clinical management of fertility issues in the context of cancer care. ${ }^{18}$ Ideally a well-connected network of oncologists, fertility specialists and psychologists is required to address fertility preservation and reproductive health issues before and after the cancer treatment. The Fertility Consortium established at the North Western University, Chicago, USA $<$ http://oncofertility. northwestern.edu/ $>$ is a good example of interdisciplinary initiative designed to explore the reproductive future of cancer survivors.

\section{Management of adolescent cancer patients}

In adolescent cancer patients, screening for male gonadal functions should be carried out annually. The screening should consist of age-appropriate history with specific attention to growth, pubertal development and problems with libido, impotence, or fertility. ${ }^{4}$ About $85 \%$ of the testicle is germinal tissue, so a reduced testicular size and soft consistency are indicative of decreased germinal tissue. Spermatogenesis can be verified directly by semen analysis or indirectly by serum levels of FSH and LH. An abnormal semen analysis is suggestive of either germ cell damage, ejaculatory dysfunction or hormone deficiencies. The male survivors of childhood cancer have a greater than 2.6-fold increased risk for erectile dysfunction. It is important that care giver pay attention to cancer survivor's sexual health and make efforts for early detection and intervention. ${ }^{28}$ 


\section{Post cancer assisted reproductive technology results}

The live birth rate using assisted reproductive technologies among cancer patients is at least comparable to that of the non-cancer population. ${ }^{29}$ Studies on women who conceived spontaneously many years after chemotherapy treatment found no statistically significant increase in congenital malformations or malignant neoplasms in the resulting offspring, ${ }^{7}$ or after assisted reproduction. ${ }^{30}$ However, if the patient has an inheritable cancer, technique of prenatal genetic testing provides a way to prevent transmission of that risk to offspring.

The use of advanced technologies of assisted reproduction like ICSI, ${ }^{31}$ PICSI, ${ }^{32}$ calcium ionophore activation ${ }^{33}$ and sperm motility inducers, ${ }^{34}$ enhances successful pregnancies using extremely poor quality fresh or cryopreserved sperm. The granulocyte colonystimulating factor promotes proliferation of undifferentiated spermatogonia, leading to a modest enhancement of spermatogenic regeneration from surviving spermatogonia after high-dose alkylating chemotherapy. ${ }^{35}$

It is recommended that the cancer survivor with severe oligozoospermia (sperm counts $\leq 5 \times 10^{6} / \mathrm{mL}$ ) whose attempts to achieve pregnancy have been unsuccessful for 6 months or more, should be referred to a male reproductive health specialist for detailed counselling or consideration of sperm cryopreservation if not already performed regardless of sperm count. After one year of failed attempts to achieve pregnancy, couple should seek help from fertility specialist. A pre-pubertal patient who has no signs of puberty by 14 years of age or who shows failure of pubertal progression referral to a pediatric endocrinologist is recommended. ${ }^{36}$

\section{Conclusion}

Cancer survival rate has increased significantly; however, cancer and its treatment adversely affect fertility and sexual life of the survivors. Cancer is affecting children, adolescents and adults. Therefore, cancer treatment providers should train their staff to meet needs of each patient category and address not only the cancer treatment but also the fertility, sexual health and post cancer life quality. Ideally a well-connected network of oncologists, fertility specialists and psychologists is required to address fertility preservation and to overcome reproductive health challenges before and after the cancer treatment.

\section{Acknowledgments}

I acknowledge support of my family for allowing me enough time to prepare this manuscript.

\section{Funding}

None.

\section{Conflicts of interest}

Authors declare that they have no competing interests.

\section{References}

1. De Sanctis V, Soliman AT, Yassin MA, et al. Testicular damage in children and adolescents treated for malignancy: a short review. Acta bio-medica: Atenei Parmensis. 2018;89(3-S):7-17.

2. Du Plessis SS, Gokul S, Agarwal A. Semen hyperviscosity: causes, consequences, and cures. Front Biosci (Elite Ed). 2013;5:224-231.

3. Sakkas D, Ramalingam M, Garrido N, et al. Sperm selection in natural conception: what can we learn from Mother Nature to improve assisted reproduction outcomes? Hum Reprod Update. 2015;21(6):711-726.
4. WHO laboratory manual for the examination and processing of human semen. 5th ed. World Health Organization; 2010.

5. Sukhu T, Ross S, Coward RM. Urological survivorship issues among adolescent boys and young men who are cancer survivors. Sex Med Rev. 2018;6(3):396-409.

6. van Dorp W. Determinants of gonadal function after childhood cancer. Doctoral thesis. 2014. 28 p.

7. Ethics Committee of the American Society for Reproductive Medicine. Fertility preservation and reproduction in patients facing gonadotoxic therapies: an Ethics Committee Opinion. Fertil Steril. 2018;110(3):380 386.

8. Mortimer D. Current and future concepts and practices in human sperm cryobanking. Reproductive Biomedicine Online. 2004;9(2):134-151.

9. Gertosio C, Magistrali M, Musso P, et al. Fertility preservation in pediatric oncology patients: new perspectives. J Adolesc Young Adult Oncol. 2018;7(3):263-269.

10. Al Fayyad IN. Oncofertility challenges in saudiarabia: ethical and religious implications. International Journal of Information Research and Review. 2016;3(6):2476-2483.

11. Ukita Y, Wakimoto Y, Sugiyama Y, et al. Fertility preservation and pregnancy outcomes in adolescent and young adult male patients with cancer. Reproductive Medicine and Biology. 2018;17(4):449-453.

12. Williams IV DH. Sperm banking and the cancer patient. Ther Adv Urol. 2010;2(1):19-34.

13. Agarwal A, Sidhu RK, Shekarriz M, et al. Optimum abstinence time for cryopreservation of semen in cancer patients. J Urol. 1995;154(1):86-88.

14. Polcz TE, Stronk J, Xiong C, et al. Optimal utilization of cryopreserved human semen for assisted reproduction: recovery and maintenance of sperm motility and viability. J Assist Reprod Genet. 1998;15(8):504-512.

15. Thomson LK, Fleming SD, Barone K, et al. The effect of repeated freezing and thawing on human sperm DNA fragmentation. Fertil Steril. 2010;93(4):1147-1156.

16. Vakalopoulos I, Dimou P, Anagnostou I, et al. Impact of cancer and cancer treatment on male fertility. Hormones. 2015;14(4):579-589.

17. Klosky JL, Anderson LE, Russell KM, et al. Provider influences on sperm banking outcomes among adolescent males newly diagnosed with cancer. $J$ Adolesc Health. 2017;60(3):277-283.

18. Benedict C, Thom B, Kelvin J. Fertility preservation and cancer: challenges for adolescent and young adult patients. Curr Opin Support Palliat Care. 2016;10(1):87-94.

19. Galuppo AG. Spermatogonial stem cells as a therapeutic alternative for fertility preservation of prepubertal boys. Einstein (Sao Paulo). 2015;13(4):637-379.

20. Ibtisham F, Wu J, Xiao M, et al. Progress and future prospect of in vitro spermatogenesis. Oncotarget. 2017;8(39):66709.

21. Ho WL, Bourne H, Gook D, et al. A short report on current fertility preservation strategies for boys. Clin Endocrinol. 2017;87(3):279-285.

22. Picton HM, Wyns C, Anderson RA, et al. A European perspective on testicular tissue cryopreservation for fertility preservation in prepubertal and adolescent boys. Human Reproduction. 2015;30(11):2463-2475.

23. Pauli C, Hopkins BD, Prandi D, et al. Personalized in vitro and in vivo cancer models to guide precision medicine. Cancer Discov. 2017;7(5):462-477.

24. Cimino GD, Pan CX, Henderson PT. Personalized medicine for targeted and platinum-based chemotherapy of lung and bladder cancer. Bioanalysis. 2013;5(3):369-391.

25. Miller KD, Siegel RL, Lin CC, et al. Cancer treatment and survivorship statistics, 2016. CA Cancer J Clin. 2016;66(4):271-289. 
26. Scherz N, Bachmann-Mettler I, Chmiel C, et al. Case management to increase quality of life after cancer treatment: a randomized controlled trial. BMC Cancer. 2017;17(1):223.

27. Pereira N, Schattman GL. Fertility preservation and sexual health after cancer therapy. J Oncol Pract. 2017;13(10):643-651.

28. Ritenour CW, Seidel KD, Leisenring W, et al. Erectile dysfunction in male survivors of childhood cancer-a report from the childhood cancer survivor study. J Sex Med. 2016;13(6):945-954.

29. García A, Herrero MB, Holzer H, et al. Assisted reproductive outcomes of male cancer survivors. J Cancer Surviv. 2015;9(2):208-214.

30. Gunnes MW, Lie RT, Bjørge T, et al. Reproduction and marriage among male survivors of cancer in childhood, adolescence and young adulthood: a national cohort study. Br J Cancer. 2016;114(3):348-356.

31. Palermo GD, Neri QV, Schlegel PN, et al. Intracytoplasmic sperm injection (ICSI) in extreme cases of male infertility. PLOS One. 2014;9(12):e113671.
32. Erberelli RF, Salgado RM, Pereira DH, et al. Hyaluronan-binding system for sperm selection enhances pregnancy rates in ICSI cycles associated with male factor infertility. JBRA Assisted Reproduction. 2017;21(1):2.

33. Ebner T, Maurer M, Oppelt P, et al. Healthy twin live-birth after ionophore treatment in a case of theophylline-resistant Kartagener syndrome. $J$ Assist Reprod Genet. 2015;32(6):873-877.

34. Ebner T, Shebl O, Mayer RB, et al. Healthy live birth using theophylline in a case of retrograde ejaculation and absolute asthenozoospermia. Fertil Steril. 2014;101(2):340-343.

35. Kotzur T, Benavides-Garcia R, Mecklenburg J, et al. Granulocyte colonystimulating factor (G-CSF) promotes spermatogenic regeneration from surviving spermatogonia after high-dose alkylating chemotherapy. Reprod Biol Endocrinol. 2017;15(1):7.

36. Skinner R, Mulder RL, Kremer LC, et al. Recommendations for gonadotoxicity surveillance in male childhood, adolescent, and young adult cancer survivors: a report from the International Late Effects of Childhood Cancer Guideline Harmonization Group in collaboration with the PanCareSurFup Consortium. Lancet Oncol. 2017;18(2):e75-e90. 